\title{
Experimental Evaluation of the Coefficient of Thermal Expansion of Chilled Aluminum Alloy-Borosilicate Glass (P) Composite
}

\author{
Anupama Hiremath ${ }^{1 *}$, Joel Hemanth ${ }^{2}$ \\ 1. Department of Mechnical\& Manufacturing Engineering, Manipal Institute of Technology, Manipal University, Manipal, \\ India, 576104 \\ 2. Department of Mechanical Engineering, H.M.S. Institute of Technology, Tumkur, Karnataka, India
}

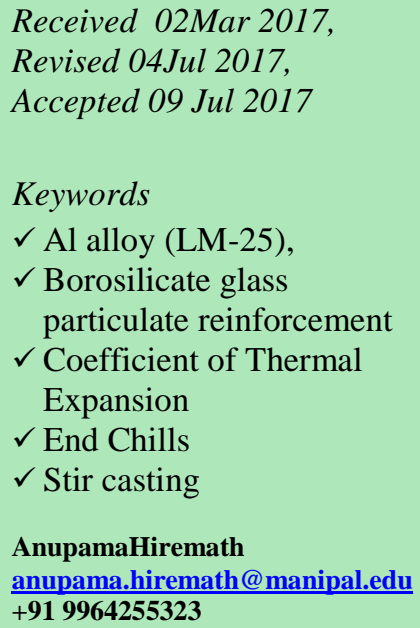

Received 02Mar 2017,

Revised 04Jul 2017

Accepted 09 Jul 2017

Keywords

$\checkmark \mathrm{Al}$ alloy (LM-25),

$\checkmark$ Borosilicate glass particulate reinforcement

$\checkmark$ Coefficient of Thermal

Expansion

$\checkmark$ End Chills

$\checkmark$ Stir casting

AnupamaHiremath

anupama.hiremath@manipal.edu $+919964255323$

\begin{abstract}
Aluminum metal matrix composites (AMCs) are fast emerging as the new generation material that are on a very brisk rate eliminating the use of conventional monolithic metals and alloys in aerospace, automobile and electronic-packing applications. Such, high technology applications calls for materials which possess near zero coefficient of thermal expansion (CTE) at higher operating temperatures. The paper investigates the coefficient of thermal expansion of the chilled LM-25 aluminum matrix composite reinforced with borosilicate glass powder. The composites are prepared via stir casting route by varying the weight percent (wt.\%) of reinforcing particulates in the metal matrix starting from 3 wt.\% and going up till 12 wt.\% with an increment of $3 \mathrm{wt} . \%$ in every step. In order to ensure sound, defect free castings, judicious use of two metallic and two nonmetallic end chills in a sand mold have been made. The CTE of the specimens drawn from the castings have been obtained. The analysis of the results illustrates a strong relationship between the CTE and wt.\% reinforcement. It is also evident that different chill materials possessing different Volumetric Heat Capacities (VHC) also bear a strong effect not only on the quality of the castings produced but also on the CTE of the AMCs.
\end{abstract}

\section{Introduction}

Composites, known for their tailorable properties, dominate the field of materials not only for their superior structural properties, but also due to their enhanced thermal, electrical and tribological properties [1]. Metal Matrix Composites (MMCs) in general and Aluminum Matrix Composites (AMCs) in particular, possess desirable properties such as high strength, specific stiffness, and hardness coupled with high resistance to wear and corrosion. They are also known for their exceptional ability to perform effectively in very high temperature applications. Due to these superior mechanical, chemical and thermal properties, AMCs find their extensive use in applications spanning from the very basic structural to the most advanced aerospace and automotive applications [2].

The dedicated research over the last few years has resulted in the emergence of discontinuously reinforced Aluminum Matrix Composites as they possess relatively better isotropic properties in comparison to continuous fiber-reinforced composites. These materials have garnered a widespread attention of both the producers as well as researchers around the world due to their exceptional strength-to-weight ratio and can be applied in high temperature and corrosive applications. These are also attractive due to their ease of manufacture providing scope for mass production and are thus believed to be economical when compared with continuously reinforced aluminum matrix composites [3].

Composites are greatly influenced by the choice of the reinforcement and its distribution within the matrix and also with the chemical affinity that the reinforcing particles possess with the matrix metal. Based on the type of the reinforcement selected, the fabrication methods can vary considerably [4-5]. But, it is a well-documented fact that liquid processing method such as stir casting and liquid infiltration techniques are the most widely used for the fabrication of discontinuously reinforced aluminum matrix composites. Since, even distribution of dispersoid within the matrix is of paramount interest, it is suggested by many investigators, that stircasting is most suitable for producing discontinuously reinforced aluminum matrix composites [6]. 
Aluminum and its alloys are difficult to feed during the casting process as they freeze over a widerange of temperatures. The dispersed porosity in the resultant castings caused by such pasty type of solidification can be effectively countered with the help of chilling during solidification [7-8]. End chills, when placed judiciously within the mold cavity promote directional solidification of the melt, thereby ensuring a sound, defectfree composites [9].

\section{Materials and methods}

\subsection{Composition of MMCs}

LM-25 aluminum alloy is a casting friendly alloy and possesses excellent mechanical and thermal properties coupled with a high resistance to corrosion [10]. LM-25 is commercially available under various conditions of heat treatments and thus is extensively used in applications such as the production of engine cylinder blocks, cylinder heads, wheels and other such automotive applications. Table 1 below illustrates the chemical composition of LM-25 aluminum alloy.

Borosilicate glass is commercially marketed under trade names such as Pyrex ${ }^{\circledR}$ and Duran ${ }^{\circledR}$. Borosilicate glass is chemically resistant, has a low thermal expansion coefficient and can be used at relatively high temperatures. It is widely used in chemical and engineering applications.

In the present work, Borosilicate glass powder is used to reinforce LM-25 aluminum alloy matrix. The size of the borosilicate glass powder is $100 \mu \mathrm{m}$ which is dispersed within the matrix material. The LM-25 aluminum alloy matrix is reinforced with 3, 6, 9 and 12 weight percent (wt.\%) of borosilicate glass powder. Table 2 depicts some of the physical and thermal properties of the borosilicate glass.

Table 1: Chemical composition of LM-25 aluminum alloy

\begin{tabular}{|l|r|l|l|l|l|l|l|}
\hline Elements & $\mathrm{Zn}$ & $\mathrm{Mg}$ & $\mathrm{Si}$ & $\mathrm{Cu}$ & $\mathrm{Mn}$ & $\mathrm{Fe}$ & $\mathrm{Al}$ \\
\hline Composition (wt. \%) & 0.10 & 0.35 & 7.0 & 0.2 & 0.1 & 0.2 & Balance \\
\hline
\end{tabular}

Table 2: Thermo-physical properties of borosilicate glass

\begin{tabular}{|c|c|c|c|c|}
\hline Property & $\begin{array}{c}\text { Density } \\
\mathrm{g} / \mathrm{cm}^{3}\end{array}$ & $\begin{array}{c}\text { Young's Modulus } \\
\mathrm{GPa}\end{array}$ & $\begin{array}{c}\text { Thermal Conductivity } \\
\mathrm{W} / \mathrm{m} . \mathrm{K}\end{array}$ & $\begin{array}{c}\text { Co-Efficient of Linear Expansion } \\
10^{-6} /{ }^{\circ} \mathrm{C}\end{array}$ \\
\hline Value & 2.23 & 64 & 1.14 & 3.3 \\
\hline
\end{tabular}

\subsection{End Chills}

Chills are specific objects employed for the purpose of extraction of heat at a fairly faster rate from the melt, thus ensuring directional solidification of the melt in the casting mold. Formation of pasty zones in sand molds pose problems in obtaining a sound casting. This problem can be resolved by establishing a steep temperature gradient during solidification which can be achieved by the incorporation of chills which act as an effective heat sink [10]. The volumetric heat capacity (VHC) of the chill material is an important parameter that defines the efficiency of the chill.

$$
\mathbf{V H C}=\mathbf{V} \times \mathbf{C}_{\mathbf{p}} \times \mathbf{\rho}-----------(1)
$$

where $\mathrm{V}$ is the volume of the chill, $\mathrm{C}_{\mathrm{p}}$ is the specific heat of the chill material and $\rho$ is the density of the chill material.

In the present research work, two metallic chills (copper and mild steel) and two nonmetallic chills (silicon carbide and graphite) have been used to produce the required LM25-borosilicate glass particulate composites. The end chills are strategically placed within the mold cavity for the promotion of directional solidification of the melt which in turn would ensure sound castings. Figure 1 illustrates the four different end chills selected for the work. The selection of the chills is based on their values of VHCs. Table 3 illustratesthe values of VHC and other thermo-physical properties for the selected end chill materials.

\subsection{Stir Casting}

Stir casting is a novel method used for the production of particulate reinforced metal matrix composites as stirring ensures proper distribution of the dispersoid within the matrix. Mechanical stirring is required to combine the two phases of a composite as most of the reinforcements exhibit poor wettability with the metal matrix. The vortex method is the most widely used method in stir casting for the dispersion of dispersoid in the molten metal. This is achieved by the vigorous stirring of the melt to produce a vortex and into this vortex the reinforcing particles are added for proper distribution within the melt. Figure 2 illustrates a typical stir casting method. 
Table 3: Thermo-physical properties of end chills

\begin{tabular}{|c|c|c|c|c|}
\hline $\begin{array}{c}\text { Material of the } \\
\text { chill }\end{array}$ & Density (g/cc) & $\begin{array}{c}\text { Specific heat } \\
(\mathrm{J} / \mathrm{kg} \mathrm{K})\end{array}$ & $\begin{array}{c}\text { Thermal conductivity } \\
(\mathrm{W} / \mathrm{m} \mathrm{K})\end{array}$ & $\begin{array}{c}\text { VHC for 25 mm chill } \\
(\mathrm{J} / \mathrm{K})\end{array}$ \\
\hline Copper & 8.96 & 0.448 & 1.022 & 597.0 \\
\hline Steel & 7.85 & 0.421 & 0.109 & 491.5 \\
\hline Cast iron & 7.61 & 0.401 & 0.160 & 453.9 \\
\hline Silicon carbide & 2.36 & 1.095 & 0.039 & 384.3 \\
\hline
\end{tabular}
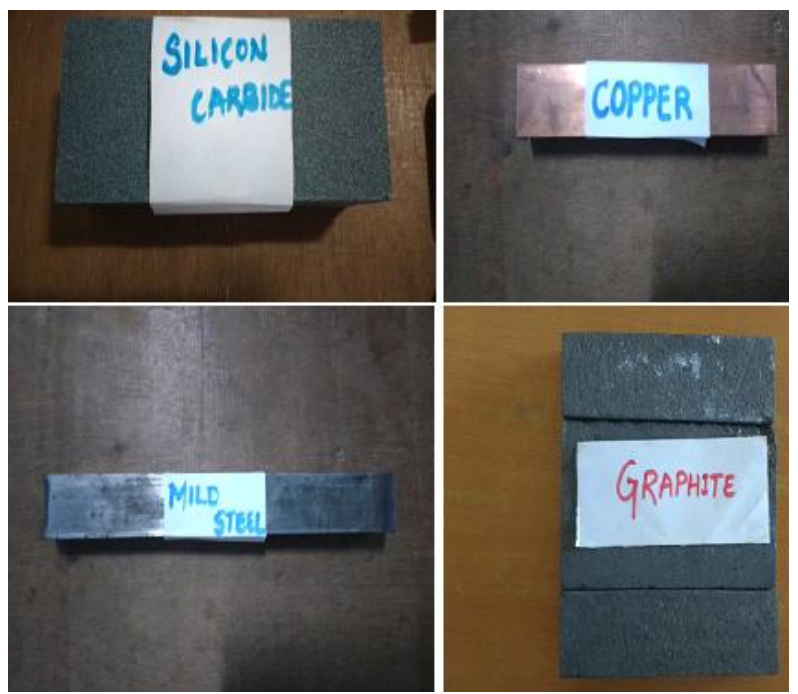

Figure1: Metallic and Non-metallic End Chills

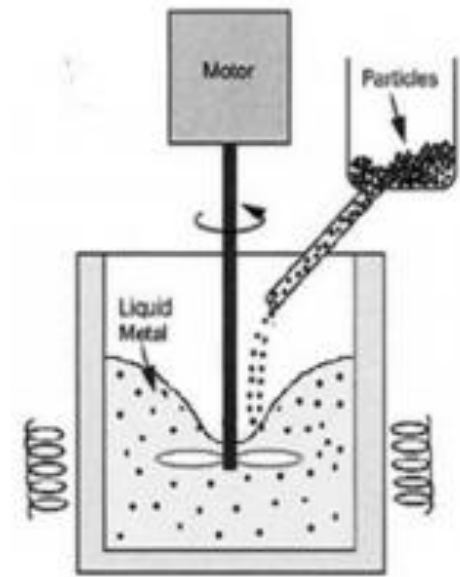

Figure 2: Stir CastingSetup

The LM-25 aluminum alloy is melt in a furnace to about $750^{\circ} \mathrm{C}$ in an inert atmosphere. The borosilicate glass powder is preheated to a temperature of $700^{\circ} \mathrm{C}$ to avoid the thermal mismatch between the matrix and dispersoid and also to drive away any volatile particles present in the reinforcing phase. The molten alloy is then stirred vigorously with the help of a motor-driven stirrer to create a vortex. The preheated dispersoid is then poured into the vortex of the melt.

\subsection{Chill Casting}

The sand molds for obtaining rectangular ingots measuring $150 \times 20 \times 20 \mathrm{~mm}$ was prepared using green foundry sand to which 5 weight percent bentonite and 5 weight percent moisture was added in accordance with AFS standards. The molds are prepared in such a manner so as to accommodate suitably, the end chills within the mold cavity. Four such molds are prepared into which the copper, mild steel, silicon carbide and graphite end chills are inserted. The end chill are rectangular blocks of $150 \times 20 \times 25 \mathrm{~mm}$ dimension. Figure 3 depicts the mold box with an end chill inserted next to the mold cavity into which the melt is to be poured. 
The melt is next poured into a sand mold cavity with a chill attached to it at one end. Different molds are prepared with different chill materials like copper, steel, iron and silicon carbide. The composite castings obtained are shown in figure 4.

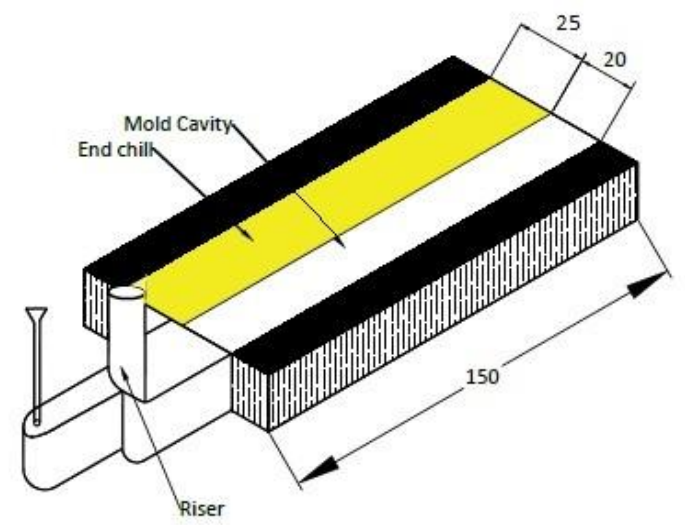

Figure 3: Mold Cavity with an end chillFigure

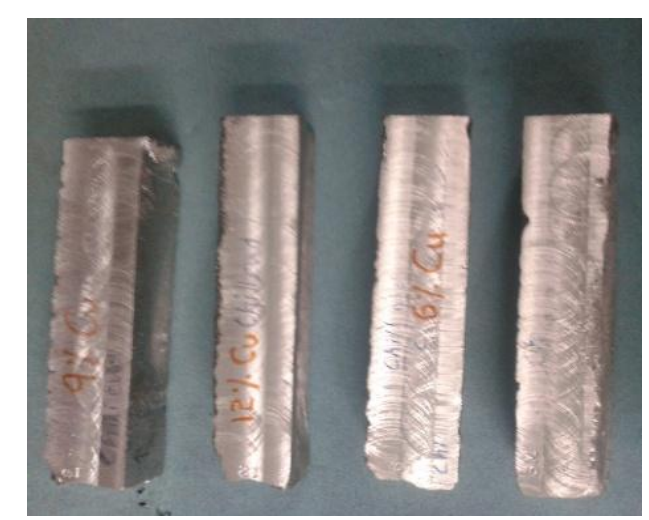

4: Cast MMCs with the aid of copper chill

\subsection{Coefficient of thermal expansion}

Composite materials are sensitive to temperature changes as the response of the matrix material to an applied load is often directly dependent on the temperature and any small changes in temperature causes the differential thermal contraction and expansion of the constituent phases that in turn results in building up of internal stresses within the material [11-12]. Thus, the study of coefficient of thermal expansion of metal matrix composites is of paramount importance.

In the present research work, Thermo-Mechanical Analyzer (TMA Q400) is used to determine the coefficient of thermal expansion of the fabricated MMCs. The TMA works on the principle of change in the dimension of the test sample with respect to temperature. Figure 5 illustrates TMA Q400 equipment used for determining CTE of the fabricated MMCs. TMA Q400 operates over a temperature range of $-150^{\circ} \mathrm{C}$ to $1000^{\circ} \mathrm{C}$ using heating rates up to $200^{\circ} \mathrm{C} / \mathrm{min}$. It determines changes in sample properties resulting from changes in four experimental variables: temperature, force, atmosphere, and time.

The rectangular specimens measuring $10 \times 5 \times 5 \mathrm{~mm}$ are machined near the chill-end of the MMCs and are polished with the help of emery paper. The specimens are then loaded onto the stage. The furnace is turned on and an expansion probe is used to measure the change in length of the specimen with respect to the temperature.

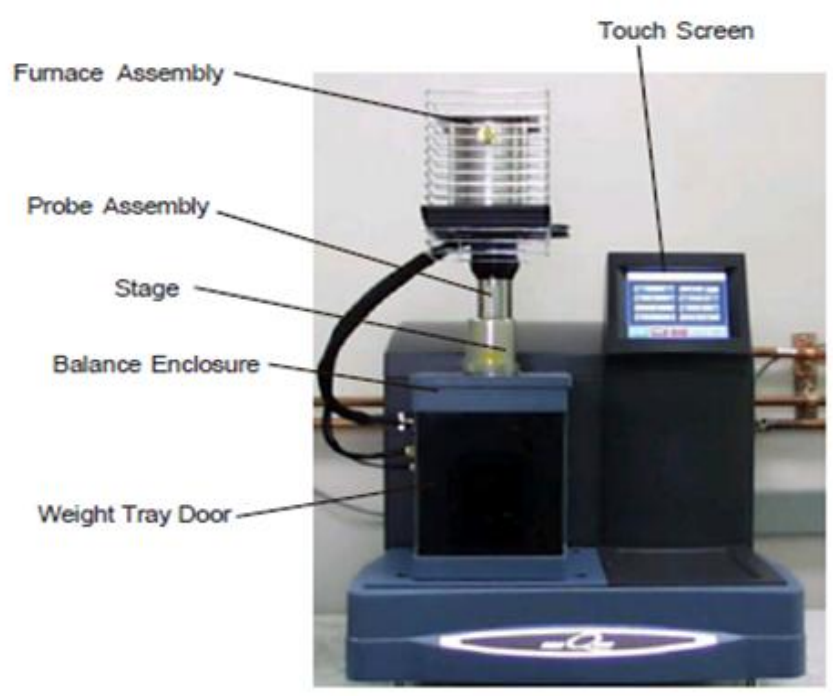

Figure 5: TMA Q400 equipment

\subsection{Microstructure examination}

Microstructural examination of the cast composites are made to evaluate the effect of chills on the microstructure of the specimens and to ascertain the chill casting process for obtaining sound, defectfree castings. The specimens are obtained from the chillend of the castings. The specimens thus obtained are 
polished first on a 600 grit emery paper followed by a second polishing on 1000 grit emery. This was followed by etching with the help of Keller's reagent to obtain a fine reflective mirror finish. The polished specimens are set in a wax mold for study under an optical microscope as shown in figure 6 . The microstructure of the specimens under different magnification are obtained and analyzed.
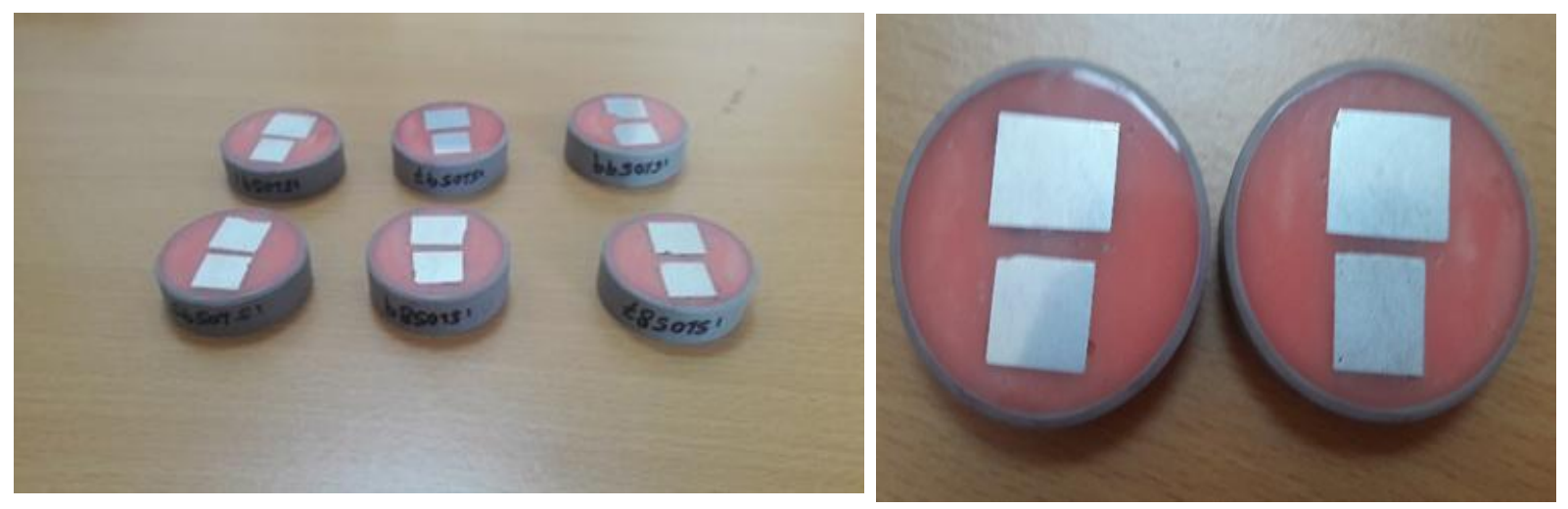

Figure 6: Specimens mounted on wax for microstructural examination

\section{Results and discussion}

\subsection{Microstructure of the MMCs}

One of the objective of the present work is to evaluate and ascertain the effect of end chills on the quality of the castings. Figure 7 (a) to 7(d) illustrates the pictomicrographs of the fabricated LM-25 aluminum matrixcomposites reinforced with $9 \mathrm{wt} \%$ borosilicate glass powder reinforcement using two metallic (Copper and Mild Steel) and two nonmetallic (Graphite and Silicon Carbide) chill.

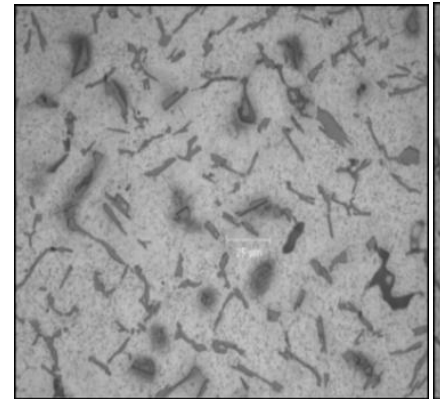

(a)

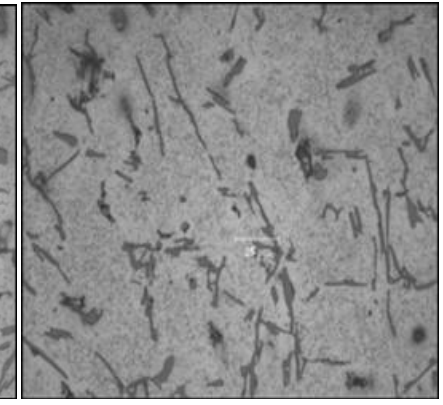

(b)

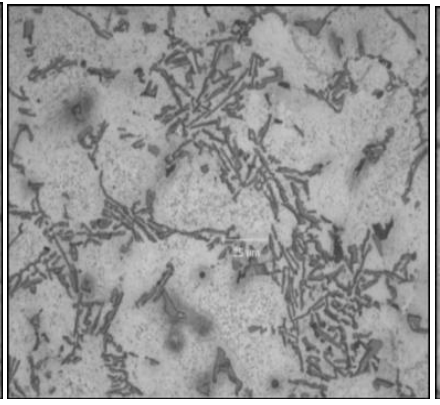

(c)

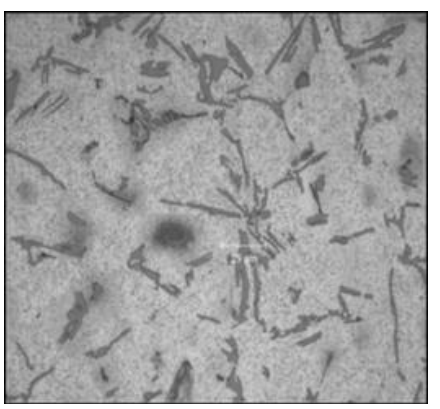

(d)

Figure 7:Microstructure of MMCs (9 wt \% reinforcement) fabricated with the help of different end chills ((a) Copper Chill (b) Mild steel Chill (c) Silicon Carbide Chill (d) Graphite Chill)

The microstructure reveals a refined grain structure for MMCs fabricated using copper and mild steel metallic chills as a steep solidification rate promotes rapid nucleation and growth of the particles resulting in a fine grain structure. Due to the chilling effect, the primary $\alpha$-Al phase cannot grow up to a dendritic structure earlier to the total solidification of the molten melt. The microstructure of the MMCs fabricated with the help of nonmetallic chills shows dendrites of primary Al with a secondary dendrite arm spacing of the order $25 \mu \mathrm{m}$. Fine precipitates of the alloy material with needle shaped eutectic silicon can also be seen in the microstructure. The microstructure also reveals a uniform distribution of the dispersoid within the matrix metal which is the result of stirring process.

The solidification of the melt under the effect of chills results in a super cooling of the melt due to intimate contact between the chill and the melt [13-14]. Under such circumstances, the critical nucleus size of the solidifying melt is reduced resulting in the generation of large number of smaller nuclei. This contributes to the generation of finer microstructure. This rapid cooling of the melt will not provide sufficient time for the dispersoid to settle down owing to the density difference between the reinforcement $\left(2.23 \mathrm{~g} / \mathrm{cm}^{3}\right)$ and the matrix metal $\left(2.7 \mathrm{~g} / \mathrm{cm}^{3}\right)$. This allows for an uniform distribution of the reinforcement within the matrix resulting in a refined microstructure with improved mechanical properties of the chilled composites. 


\subsection{Coefficient of thermal expansion (CTE)}

The dimensional stability of the composite is greatly influenced by CTE. The variation of CTE in MMCs is dependent on their respective dispersoid content, shape and orientation of the dispersoid within the matrix [15]. Table 4 and figure 8 gives the CTE values obtained at different temperatures for the fabricated chilled AMCs with varied weight $\%$ particulate content.

Table 4: Coefficient of thermal expansion of Al-borosilicate glass powder chilled composite for different wt.\% of dispersoid tested at different temperatures

\begin{tabular}{|c|r|r|r|r|}
\hline \multirow{5}{*}{ Temperature $\left({ }^{\circ} \mathrm{C}\right)$} & \multicolumn{4}{|c|}{ Dispersoid content (wt.\%) } \\
\cline { 2 - 5 } & \multicolumn{1}{|c|}{3} & \multicolumn{1}{c|}{6} & \multicolumn{1}{c|}{9} & \multicolumn{1}{c|}{12} \\
\hline 50 & 22.3 & 21.69 & 20.1 & 18.61 \\
\hline 100 & 22.71 & 22.06 & 19.23 & 19.1 \\
\hline 150 & 23.34 & 22.47 & 21.11 & 20.06 \\
\hline 200 & 24.01 & 23.18 & 21.9 & 20.81 \\
\hline 250 & 24.87 & 23.61 & 22.62 & 22.01 \\
\hline
\end{tabular}

The tabulated results clearly establishes a linear relationship between the CTE of the fabricated MMC and the weight $\%$ of the reinforcing phase. The CTE decreased linearly with increased particulate content. At $100^{\circ} \mathrm{C}$, the reduction in CTE for $3 \mathrm{wt} . \%$ reinforcement is around $39.73 \%$ less than that of the matrix aluminum alloy, the CTE of which is around $37 \mu \mathrm{m} / \mathrm{m}^{\circ} \mathrm{C}$ at $100^{\circ} \mathrm{C}$. The difference in CTE at $100^{\circ} \mathrm{C}$, in comparison to thealuminum alloy, is about $48.38 \%$ less with $12 \mathrm{wt} . \%$ reinforcement being added to the matrix alloy. This trend clearly shows that the expansion of soft aluminum alloy is captured by hard borosilicate glass particulates, thus bringing down the total CTE of the MMCs.

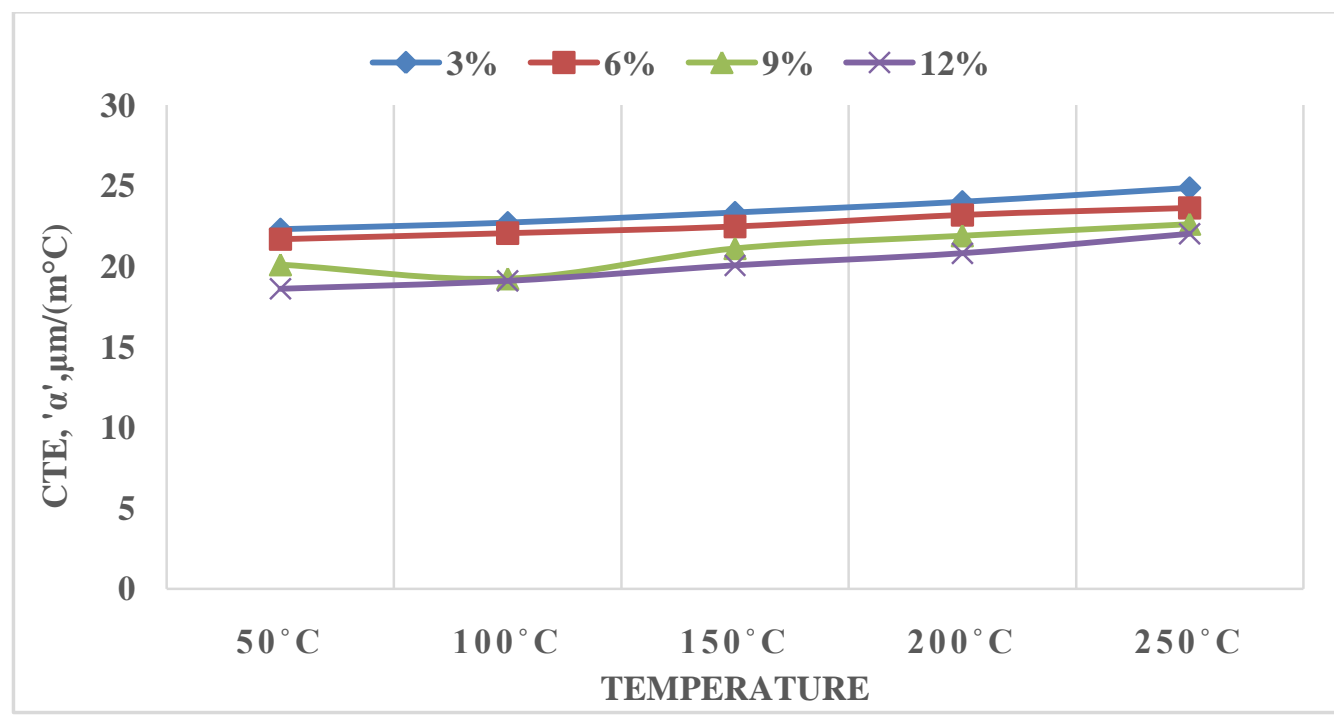

Figure 8: Coefficient of thermal expansion of Al-borosilicate glass powder chilled composite for different wt.\% of dispersoid tested at different temperatures

The lower CTE of the composites can also be attributed to a proper distribution of particles within the matrix which is evident from the microstructural examinations. The variation in CTE of a MMC largely depends on its microstructure [16-17]. A uniform distribution of dispersoid within the matrix material results in a better thermal and mechanical properties, whereas, the presence of voids within the microstructure would severely hamper the thermo-mechanical properties of the metal matrix composites [18]. The CTE of a particular MMC with a fixed wt. \% of reinforcement increases with temperature [19] as seen in figure 8. This is due to plasticization of matrix alloy which would in turn results in filling up of micro voids and eventually taking the CTE to a higher value. The reduction of CTE is also due to the presence of borosilicate glass powder which has a relatively low CTE value as compared to the LM-25 aluminum alloy. 


\section{Conclusions}

Employment of end chills with higher volumetric heat capacities for casting aluminum matrix composites would take care of the solidification problems that are encountered while casting aluminum and its alloys. Thus, chills are an effective and economical means for producing sound, defectfree aluminum matrix composite castings. Also, stirring the melt while adding the reinforcement particles ensures proper and uniform distribution of particles within the melt. The CTE of the cast composites reduces with increasing the weight percent of borosilicate glass powder reinforcement within the matrix. Thus, adding low CTE reinforcement to high CTE metal matrix can drastically bring down the CTE of composites.

\section{References}

1. Rajkumar, K., Aravindan, S. CompositeMaterials(2017)433-455.

2. Joel Hemanth, Materials and Design, 30 (2009) 323-329.

3. Mohan Krishna S. A., Shridhar Dr. T N, Krishnamurthy Dr. L, Int. J. Mod.Engg. Res., 4 (8) (2014)53-62.

4. Tabish Qamar Hashmi, Int. J. Res. Mech. Engg. Tech., 5(1) (2014) 103-106.

5. Youssef Y., El-Sayed M., Int. Rev. Mech. Engg. (IREME), 10 (4) (2016) 261-265.

6. Karvanis K., D. Fasnakis A. Maropoulos, and S. Papanikolaou, IOP Conference Series: Mat. Sci. Engg., 161(1) (2016) 012070, doi:10.1088/1757-899X/161/1/012070.

7. Hemanth J., J. Chem. Engg. Mat. Sci., 2(8) (2011) 110-121.

8. Wang Dong Tao, Hai Tao Zhang, Ke Qin, Xing Han, Bo Shao, Ke Sheng Zuo, Jian Zhong Cui. Materials Science Forum, 877 (2017) 15-19.

9. Hiremath A., Hemanth J., Int. J. App. Engg. Res., 10(20) (2015) 41685-41688.

10. Ramnath B.V., Elanchezhian C., Jaivignesh M, Rajesh S, Parswajinan C, Ghias AS, Materials \& Design, 58(2014) 332-338.

11. Yan Ji, Jean E. Marshall, Eugene M. Terentjev, Polymers, 4 (2012) 316-340.

12. Shirasu K., Nakamura A., Yamamoto G., Ogasawara T., Shimamura Y., Inoue Y., Hashida T., Composites Part A: Applied Science and Manufacturing 95 (2017) 152-160.

13. Joel Hemanth, Modeling and Numerical Simulation of Material Science, 7 (2017) 1-18.

14. Goto Ikuzo, SetsuoAso, Mate. Trans.58.3 (2017)471-478.

15. Krishna S.A., Shridhar T.N., Krishnamurthy, L., Int. J. Engg. Sci. Mgmt. Research, 3(03) (2016) 2349-6193.

16. Ceschini L., Dahle, A., Gupta, M., Jarfors, A.E.W., Jayalakshmi, S., Morri, A., Rotundo, F., Toschi, S. Singh R.A., Aluminum and magnesium metal matrix nanocomposites. Springer.(2017)

17. Huang Lujun, Lin Geng,Discontinuously Reinforced Titanium Matrix Composites, ISBN 978-981-10-44472 (2017).

18. Kalantari M., Dong C., Davies, I. J. Composites Part B: Engineering, 123 (2017) 136-147.

19. Smart D. S., Nithin P. Johns, JosesJenish Smart, Mater. Sci. Forum, 889 (2017) 55-62.

(2017) ; http://www.jmaterenvironsci.com 